\title{
Development of the mammalian lymphatic vasculature
}

\author{
Ying Yang and Guillermo Oliver
}

Department of Genetics, St. Jude Children's Research Hospital, Memphis, Tennessee, USA.

\begin{abstract}
The two vascular systems of our body are the blood and lymphatic vasculature. Our understanding of the cellular and molecular processes controlling the development of the lymphatic vasculature has progressed significantly in the last decade. In mammals, this is a stepwise process that starts in the embryonic veins, where lymphatic EC (LEC) progenitors are initially specified. The differentiation and maturation of these progenitors continues as they bud from the veins to produce scattered primitive lymph sacs, from which most of the lymphatic vasculature is derived. Here, we summarize our current understanding of the key steps leading to the formation of a functional lymphatic vasculature.
\end{abstract}

\section{Origin and specification of lymphatic ECs}

Origin of lymphatic ECs in the mammalian embryo. The lymphatic vasculature is essential for maintaining interstitial fluid homeostasis. Deficiency in development and/or function of the lymphatic vasculature causes various lymphedema syndromes in humans, and active lymphangiogenesis plays a significant role in chronic inflammation and tumor metastasis. Research of the lymphatic vasculature dates back to the 17 th century, but the anatomic features of the developing lymphatic vasculature were most extensively characterized during the 20 th century by using pig and mouse embryos $(1,2)$. Florence Sabin first suggested that the lymphatic vessels arise from preexisting blood vessels, specifically the cardinal veins (CVs) (1). More than 100 years later, experiments using detailed lineage tracing in mammals and live imaging in zebrafish demonstrated that lymphatic EC (LEC) progenitors originate in the veins from venous ECs (VECs) (3, 4). Recent work in mouse embryos shows that the intersomitic veins (ISVs) and the superficial venous plexus are additional sources of LEC progenitors $(5,6)$. These findings support previous observations that LEC progenitors are of venous origin and that they leave the veins via specific migratory paths that extend radially from the dorsal half of the $\mathrm{CV}(7,8)$. Additionally, they help to explain the rapid appearance of large numbers of migrating LECs within the mesenchymal tissue (Figure 1).

LEC fate specification. Development of the mammalian lymphatic vasculature is a stepwise process in which LEC progenitors are first specified in the embryonic veins and then bud from the veins to form the primitive lymph sacs from which most of the lymphatic vasculature will eventually be derived (9). During the past few years, several key regulatory molecules and specific markers of the lymphatic endothelium have been identified (Table 1). The expression of the transcription factor Prox 1 in a subpopulation of VECs (referred to as LEC progenitors) in the embryonic CVs at approximately E9.5 $(3,5,7)$ is the initial step in the formation of the lymphatic vasculature. The transcription factors Sox 18 and COUP-TFII are required for activation of Prox1 expression in VECs (Figure 1 and refs. 10,11), and loss of either Sox 18 or COUP-TFII results in the absence of LEC progenitors (Tables 2, 3, and 4 and refs. 10-12).

Conflict of interest: The authors have declared that no conflict of interest exists. Citation for this article: J Clin Invest. 2014;124(3):888-897. doi:10.1172/JCI71609.
COUP-TFII mediates venous cell fate differentiation and is required for the specification of LEC progenitors. Its activity in the veins is regulated epigenetically by the chromatin-remodeling enzyme BRG1, a member of the SWI/SNF protein family (13). In the CV, COUP-TFII represses Notch activity, is a direct in vivo activator of Prox 1 during early LEC specification, and is also required to maintain PROX1 expression in LECs (11). Functional inactivation of COUP-TFII in LEC progenitors reduces the number of LECs (11). Several studies have shown that Notch signaling inhibits LEC fate and LEC sprouting in vitro $(14,15)$. Recently, it was shown to also play a role in LEC specification during embryonic development in vivo (16). Downregulation of Notch activity leads to an increased number of LEC progenitors, indicating that Notch signaling is a negative regulator of LEC specification (16). This new finding highlights the relationship between the COUP-TFII and Notch pathways in arterial-venous-lymphatic cell fate decisions (Figure 1).

Mutations in SOX18 have been identified as the cause of hypotrichosis-lymphedema-telangiectasia in humans (17), and MAPK/ ERK signaling is a newly discovered activator of Sox18 in the embryonic veins (18). RAF1 mutations that activate MAPK/ERK signaling are associated with Noonan syndrome, a disease that includes lymphangiectasia $(19,20)$.

Gain- and loss-of-function studies revealed that Prox 1 plays an important role in both the induction $(21,22)$ and the maintenance (23) of LEC fate. An early knockout mouse study revealed that loss of Prox1 led to absence of the lymphatic vasculature, as LEC fate was never acquired (7). PROX1 expression levels are also crucial for normal lymphatic vasculature formation, as Prox1-heterozygous embryos have significantly fewer LEC progenitors in their embryonic veins and all develop edema, a typical sign of lymphatic vascular dysfunction $(11,24)$. Prox1 haploinsufficiency causes perinatal death and, in most mouse strains, pups exhibit chylothorax and chylous ascites (Tables 2-4). On an NMR imaging background, $10 \%-20 \%$ of Prox 1 heterozygous mice live to adulthood; however, their lymphatic vessels are leaky, and they develop adult-onset obesity (24). Interestingly, not all PROX $1^{+}$LEC progenitors bud from the veins; a small group of those cells remain in place, where they contribute to the formation of the lymphovenous valves at the main connection between the jugular lymph sacs and the adjacent veins at approximately E11.5 (ref. 25 and Figure 2). 
A
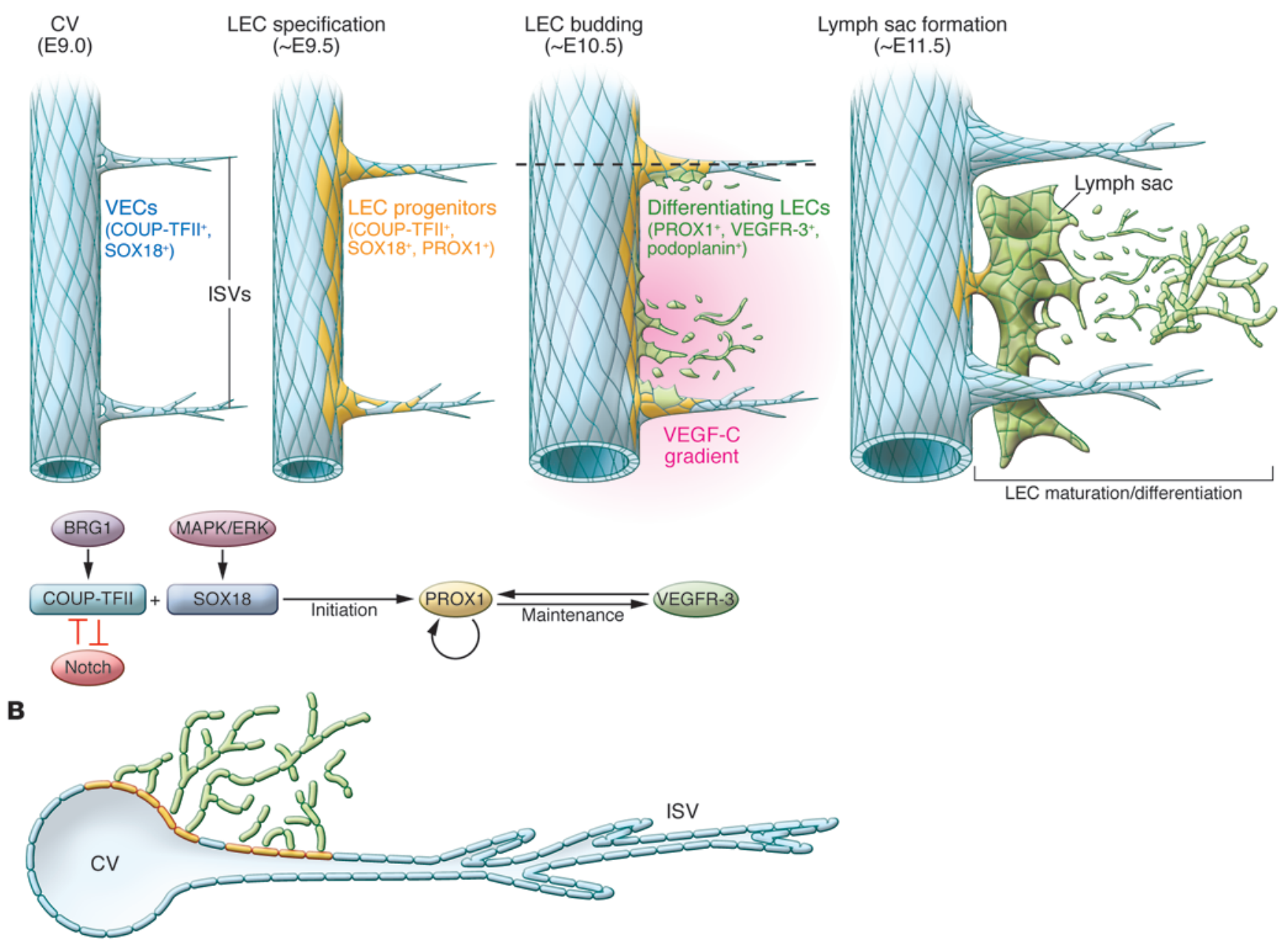

Figure 1

Schematic representation of the development of the mammalian lymphatic vasculature. (A) Sagittal view of the key temporal events that take place along the CV from E9.0 to E11.5 in the mouse embryo. The CV is the main source of LECs. Initially, among others, blood ECs in the CV and ISVs express the transcription factors COUP-TFII and SOX18. A few hours later (E9.5), the activity of SOX18 and COUP-TFII induces PROX1 expression in a subpopulation of venous ECs. The initiation of PROX1 expression indicates that LEC specification has started, and venous PROX1-expressing ECs are considered LEC progenitors. At around E10.5, most of those progenitors start to bud from the CV and ISVs. This process requires the graded expression of VEGF-C in the surrounding mesenchyme. Mediated by PROX1, budding LECs maintain the expression of VEGFR-3 and begin expressing PDPN once outside of the CV. The combined expression of these genes indicates that lymphatic differentiation is progressing. As LECs bud off in an interconnected manner, they assemble together, and at approximately E11.5 they start to form different lymph sacs. Following LEC proliferation and sprouting, the majority of the lymphatic network arises from these sacs. (B) Transverse representation of the LEC budding process. At around E10.5, PROX1+/PDPN+/VEGFR-3+-differentiating LECs bud from the CV and ISVs. The budding LECs migrate as an interconnected group of cells dorsally and longitudinally into the surrounding mesenchyme in the anterior region of the embryo.

\section{Formation of lymph sacs}

Budding of LECs from the veins. Once PROX1-expressing LEC progenitors are specified in the embryonic veins at approximately E9.5, they start to bud into the surrounding mesenchyme (Figure 1). Electron microscopy of E10.5 mouse embryos revealed that LEC progenitors bud as groups of cells interconnected by adhesion junctions expressing high levels of VE-cadherin. These junctions ensure that venous endothelial integrity is not compromised during budding (5). These results are consistent with a previous report showing that PROX $1^{+}$cells actively budding from the CVs at E12.5 are joined by zipper-like junctions expressing
VE-cadherin at their cell borders (26). When the cell-cell junction was disrupted, lymphatic vasculature development was disturbed and edema developed $(27,28)$.

Although Prox1-deficient mice are devoid of a lymphatic vasculature, initial analysis suggested that ECs were able to bud off from the CV in Prox1-null embryos (7), indicating that PROX1 is vital for the specification of LEC fate but not for LEC budding from the veins. However, a more recent evaluation using confocal microscopy of semi-thick sections of Prox1-null embryos revealed that PROX1 activity is also required for the budding of LECs from the CVs (5). This analysis showed that cells with an activated Prox1 


\section{Table 1}

Marker expression during mammalian lymphatic vasculature development

Cell type
LEC progenitor
Migrating specified LECs
Lymphovenous valves LECs
LECs in collecting lymphatics
LECs in capillaries
LECs in lymphatic valves

\section{Cell type}

LEC progenitor

Embryonic stage
E9.5-E12.5
E10.5-E12.5
E12.5-adult
E15.5-adult
E15.5-adult
E16-adult

Tissue site

Embryonic veins

Mesenchymal tissue outside the veins

Lymphovenous valves

Collecting lymphatics

Lymphatic capillaries

Collecting lymphatics

\author{
Marker expression \\ PROX1 ${ }^{\mathrm{med} / P D P N}$-/LYVE1+/SOX18+/VEGFR-3med/NRP2 ${ }^{\text {med }}$

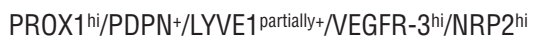 \\ PROX1 1 hi/PDPN-/LYVE1 (patchy)/NEGFR-3-/FOXC2 $2^{\text {hi } / G A T A 2+~}$ \\ PROX1+/PDPN+/LYVE1'/Reelin (extracellular)/VEGFR-310 \\ PROX1+/PDPN+/LYVE1+/Reelin (intracellular)/VEGFR-3+ \\ PROX1 ${ }^{\text {hi/FOXC2hi/LYVE1 } 10 / G A T A 2+/ L a m i n i n-~} \alpha 5^{+} /$ \\ Integrin- $\alpha 9^{+} /$VEGFR-3h/PDPN ${ }^{+}$
}

promoter are located in the ISVs rather than in the mesenchyme surrounding the CVs of Prox1-null embryos, demonstrating that LEC progenitors had failed to leave the CVs and ISVs (5).

Budding of LEC progenitors is not only associated with cell and nuclear shape change (6), but also with changes in LEC marker expression. The expression of podoplanin (PDPN) is an important molecular distinction between LEC progenitors inside the veins and those that budded. PDPN is expressed by budding PROX1expressing LECs only when they fully exit the endothelium of the embryonic veins $(5,29)$, allowing a molecular distinction of venous PROX1-expressing LEC progenitors from differentiating PROX1/ PDPN-expressing LECs that contribute to lymph sac formation outside the embryonic veins (Figure 1). The mechanism by which PDPN expression is activated in LECs remains to be elucidated. The expression pattern of PDPN suggests that it is either repressed by the venous environment or induced by factors outside the veins.

VEGF-C/VEGFR-3 signaling is required for budding of LECs from the CVs $(30,31)$, as PROX1-expressing LEC progenitors fail to detach from the CV in Vegfc-deficient mice (ref. 31 and Tables 2-4) and $\mathrm{Vegf \textrm {C } ^ { + / - }}$ embryos and adult mice develop cutaneous lymphatic hypoplasia and lymphedema (31). Conversely, overexpression of VEGF-C in the skin of transgenic mice induces selective hyperplasia of the lymphatic vasculature (32). As the primary receptor of VEGF-C, VEGFR-3 expression is restricted to LECs after E10.5 (33). Mutation of Vegfr 3 in mouse embryos demonstrated that the tyrosine kinase activity of Vegfr 3 , but not the ligand-binding domain, is essential for budding and separation of LEC progenitors from the veins (34). Heterozygous mutations in both mouse and human VEGFR-3 tyrosine kinase domains leads to lymphatic vascular defects; Chy mice display lymphedema and chylous ascites, while humans develop primary lymphedema (Milroy's disease) (35-38). In addition to VEGFR-3, VEGF-C also binds the coreceptor neuropilin-2 (NRP2) (39). NRP2 colocalizes and interacts directly with VEGFR-3 in lymphatic vessels $(39,41)$. The interaction between NRP2 and VEGFR-3 mediates proper lymphatic vessel sprouting (40). Blocking VEGF-C binding to NRP2 inhibits LEC tip cell sprouting in vivo (40). Although loss of NRP2 function does not affect the budding of LECs from the veins, the number of small lymphatic vessels and capillaries is severely reduced in NRP2 knockout mice (41).

The secreted protein CCBE1 was identified as a regulator of LEC budding in a zebrafish genetic screen (42). In that study, zebrafish embryos with mutated Ccbe1 alleles lacked a lymphatic vasculature and displayed severe edema (42). In early mouse embryos, Ccbe1 is expressed in cardiac progenitors and in mesenchyme near the nascent lymphatics $(43,44)$. When Ccbe1 is functionally inactivated in mice, the specified LEC progenitors remain in the veins and the lymphatic vasculature does not develop (Tables 2-4 and refs. 6, 44). Unlike Vegfc ${ }^{-/-}$embryos, Ccbe1 $1^{-/-}$embryos display dysmorphic sprouts and projections from the ISVs at E10.5 and E11.5, respectively (6). CCBE1 induces lymphangiogenesis by enhancing VEGF-C function independently of the tyrosine phosphorylation activity of VEGFR-3 (44). Moreover, human mutations in CCBE1 cause a type of lymphatic dysplasia known as Hennekam syndrome (45-47). Because loss of either Vegfc or Ccbe1 arrests the budding of LEC progenitors, both molecules appear to be required for this process.

Lymph sac formation. Immunostaining of semi-thick sections of E10.5-E12.5 mouse embryos showed that after LEC progenitors bud from the veins, they merge along the embryo's anterior-posterior axis to form unique intermediate structures called lymph sacs (5). At mid-gestation, the lymph sacs are the main source of LECs required for the formation of the entire lymphatic vasculature. Like the lymphatic vessels, the lymph sacs have a luminal structure comprising a single EC layer. Unlike the lymphatic vessels, mammalian lymph sacs have an irregular, sac-like shape when viewed in sagittal section (Figure 1). Because mouse embryos are relatively opaque, deep-tissue live imaging of the early steps in lymph sac formation remains technically challenging. The current model suggests that LEC progenitors bud from the veins to form lymphatic plexuses that develop into lymph sacs $(1,2)$. Recent analysis of this process by ultramicroscopy and by $3 \mathrm{D}$ reconstructed confocal images of semi-thick sections suggested that lymph sacs form by a stepwise process $(5,6)$. The initial LEC progenitors were shown to bud and migrate as an interconnected stream of cells that eventually assembled into a capillary-like structure along the anterior and posterior axes of the embryo. This structure condensed and organized to form the lymph sacs after further sprouting and migration (Figure 1 and refs. 5, 6).

Several factors that influence lymph sac formation in mammals have recently been identified (Table 1). For example, loss of any of the components of adrenomedullin signaling (adrenomedullin and its receptors Calcr1 and Ramp2) results in hypoplastic lymph sacs and subcutaneous edema (48). Mouse embryos with macrophage-specific PU.1 deficiency exhibit hypoplastic jugular lymph sacs at E14.5 (49). TIE1-deficient (angiopoietin receptor-deficient) embryos displayed enlarged lymph sacs and nuchal edema $(50,51)$. Lymph sacs in mice lacking the transcription factor $N$ fatc1 showed decreased luminal area, indicating that NFATC1 also regulates lymph sac formation (52). Lymph sacs appeared hypoplastic in Gata2-knockout embryos (53). Interestingly, mutations in GATA2 
Table 2

Loss-of-function phenotypes of genes associated with lymphatic vasculature development and disease

Gene

Loss-of-function phenotype in animal models

\section{Defective LEC progenitor specification}

Coup-TFII

Notch1

Severe subcutaneous edema; embryos lack LECs and lymphatic vasculature due to failure in LEC progenitor specification

Loss of Notch1 results in an increased number of PROX1+ LEC progenitors

in the veins and outside the CV with significant lymphatic overgrowth, incomplete separation of veins, and formation of lymphatics

Prox1 Severe subcutaneous edema; embryonic lethality at E14.5; embryos lack LECs and lymphatic vasculature due to failure in LEC progenitor specification; in most genetic backgrounds, Prox1 haploinsufficiency results in defects in LEC fate maintenance, perinatal death with chylothorax, and chylous ascites; in the NMRI strain, surviving Prox 1 heterozygous mice exhibit adult onset obesity, leaky lymphatics, and lack of lymphovenous valves

Sox18 Severe subcutaneous edema; embryonic lethal at E14.5; embryos lack LECs and lymphatic vasculature due to failure in LEC progenitor specification in certain genetic backgrounds

Defective budding of LEC progenitors and lymph sac formation

AM

AM-null embryos die at mid-gestation with interstitial edema and abnormal jugular lymphatics due to defective LEC proliferation

Calcrl Severe interstitial edema, embryonic lethal, hypoplastic lymph sacs

Ccbe1 Severe subcutaneous edema, budding of LEC progenitors arrested in the CV, lack of lymphatic vasculature

Gata2 Hypoplastic lymph sacs and abnormal separation of venous and lymphatic vessels

Nfatc1

PU.1

Ramp2

Tie1

Vegfc

Vegfr3

\section{Enlarged lymph sacs}

Hypoplastic lymph sacs, hyperplastic lymphatic vessels

Severe interstitial edema, embryonic lethal, hypoplastic lymph sacs

Reduction in TIE1 levels results in abnormal lymphatic patterning and dilated and disorganized lymphatics; homozygous null embryos are lethal at E14.5 and exhibit nuchal edema, hemorrhages, enlarged lymph sacs, dilated lymphatic vessels and impaired lymphatic drainage

Embryonic lethal at E14.5; severe subcutaneous edema, budding of LEC progenitors arrested in the CV, lack of lymphatic vasculature

Primary receptor for VEGF-C, blood vasculature malfunction in loss of function embryos, required for survival and migration of LECs
Related human vascular disease

Reference(s)

11,12

16

$7,8,23-25$

Hypotrichosis-lymphedema-

10,17 telangiectasia syndrome

\section{8}

48

Hennekam syndrome

$42,44-47$

Emberger syndrome

53,54

52

49

48

50

31

Milroy disease
$33,35,36$, 38,116 are associated with Emberger syndrome, myelodysplastic syndrome, acute myeloid leukemia, and MonoMAC syndrome with primary lymphedema $(54,55)$. GATA2 is also expressed in lymphatic valves, suggesting that it may play a role in lymphatic valve formation (55).

\section{Separation of the venous and lymphatic vasculatures}

Histologic evidence has shown that the jugular lymph sacs and jugular veins remain connected by small apertures after the lymph sacs form at approximately E12.5 $(2,56)$. Each lymph sac maintains its connection to the adjacent vein, and lymphovenous valves at the junctions of the subclavian veins and jugular lymph sacs prevent the reflux of blood into the lymphatic vessels (ref. 57 and Figure 2). Recent work molecularly characterized the formation of the lymphovenous valves, showing that they form through the intercalation of lymph sac-derived PROX $1^{+}$LECs with a previously unidentified population of $\mathrm{PROX} 1^{+} \mathrm{ECs}$ in the adjacent veins. These venous ECs correspond to a small subpopulation of PROX1-expressing ECs that do not become LEC progenitors and do not bud from the veins or acquire LEC characteristics, but instead remain within the veins (25).
Several mouse mutant models have shown defects in the separation of the lymphatic and blood vasculatures, resulting in bloodfilled lymphatic vessels (Tables $2-4$ and ref. 58). For example, mice deficient in Syk, SLP76, Runx1, or PDPN all develop blood-filled lymphatics at specific embryonic time points (3, 59-61). Interestingly, Syk, SLP76, and Runx1 are essential for hematopoiesis (62-66), which suggested that hematopoietic cells play a role in keeping the two vascular systems separated. The nature of the cell lineages involved and the mechanisms by which they control blood and lymphatic vessel separation remained unclear until several reports proposed that platelets are required for separation of the forming lymph sacs from the CVs at their connecting points (56, 67-69). In addition to being an early marker of LEC specification/ differentiation, PDPN is important in initiating platelet aggregation $(70,71)$. During the embryonic separation of lymphatics from blood vessels, LEC-expressing PDPN activates the platelet receptor CLEC2, initiating downstream SYK-SLP76 signaling. Mice lacking the megakaryocyte transcription factors Meis1 and Clec2 displayed blood-filled lymphatic vessels, further supporting the essential role of platelets in separating the blood and lymphatic vasculature dur- 
Table 3

Loss-of-function phenotypes of genes associated with lymphatic vasculature development and disease

Gene

Loss-of-function phenotype in animal models

\section{Defective lymphatic vessel maturation and valve formation}

Akt1 Reduced diameter and EC number in lymphatic capillaries; loss of valves in the smaller collecting lymphatic vessels in the superficial dermal layer of the ear skin

Bmp9 Mutant pups exhibit hyperplastic mesenteric collecting vessels with abnormally high LYVE1 expression, reduction in the number and in the maturation of mesenteric lymphatic valves

Celsr1 Disorganized cell-cell junction, defects in cell reorientation during lymphatic valve formation, lack of lymphatic valves Defects in the demarcation of the valve territory

Cnb1

CX37

CX43

CX47

Emilin1

Ephrin B2

\section{Fn1}

Foxc2

Integrin- $\alpha 9$

Nrp1

Plxna1

Reelin
Mutant mice exhibit lymphedema and chylothorax and have defective valve formation in collecting lymphatics; regulates jugular lymph sacs size Loss of lymphatic vessels in the diaphragm, absence of lymphatic valves in mesenteric collecting lymphatic vessels Expressed in lymphatic valves, currently uncharacterized developmental function Defective lymphatic valve structure and lymph flow

Hyperplastic collecting lymphatic vessels, lack of valves, abnormally high level of expression of LYVE1 in the lymphatic vessels Defects in the formation and extension of the valve leaflets Embryonic lethal perinatally; unusual mural cell recruitment on the collecting lymphatic vessels with abnormal high level of LYVE1 expression, lack of lymphatic valves, and lymph backflow Reduced number of valves leads to failure in the formation of the matrix core of the valves (leaky, lymph backflow) A mutation in the SEMA3A binding site of NRP1 leads to smaller lymphatic valves and ectopic SMC coverage on the valve region Smaller lymphatic valves

Reduced SMC coverage on the collecting lymphatic vessels with abnormally high level of LYVE1 expression, dilated and leaky collecting lymphatic vessels, and reduction in the rate of lymph flow
Related human

vascular disease

Reference(s)

79

89

89

Primary lymphedema

$89,91,92$

102

107

101

Lymphedema-distichiasis syndrome

Congenital chylothorax

101,103

106

106

80

106 ing lymphangiogenesis (67-69). It has also been shown in cultured LECs that overexpression of PDPN promotes EC migration, adhesion, and tube formation (61). A more recent proposal suggested that PDPN/CLEC2 binding could activate platelets in the gaps between the lymph sacs and the CVs, enabling blood/lymphatic vessel separation by inhibiting LEC migration, proliferation, and tube formation in the developing embryo (72). Endothelial-specific deletion of the Rho GTPase Rac1 also resulted in blood-filled lymphatics, indicating that RAC1 helps to ensure proper lymphatic-blood vessel separation by regulating LEC budding and migration (73). The cell adhesion molecule coxsackie and adenovirus receptor (CXADR) also plays an essential role in lymphatic vasculature development. CXADR is localized in cell-cell junctions in LECs, and its deletion in E12.5 mouse embryos results in embryonic death. These embryos exhibit dilated dermal lymphatic vessels, subcutaneous edema, defective cell-cell junctions in LECs, and blood-filled lymphatics (28). In addition to the blood-filled lymphatic phenotype, animals deficient in fasting-induced adipose factor (FIAF) develop gut-specific dilated intestinal lymphatic vessels and blood-filled small intestinal villi due to the defective separation of the intestinal lymphatic vasculature from the blood vasculature (74).

\section{Formation of lymphatic vessels and valves}

As the lymph sacs form, LECs continue to proliferate and migrate into the mesenchymal tissue. During this process, the primitive lymphatic plexus further differentiates to form the two distinct mammalian lymphatic vessel types: collecting lymphatic vessels (larger) and lymphatic capillaries (smaller) (75). Although precollectors have been considered a third type of lymphatic vessels, their definition is ambiguous. In general, precollectors have features of both collecting vessels and capillaries, as they lack SMC coverage but contain valves, a feature typical of collecting lymphatics. Lymphatic capillaries are thin-walled, blind-ended vessels within the tissue spaces that absorb interstitial fluid and transport it to the larger, collecting lymphatic vessels. Collecting lymphatics are surrounded by SMCs that facilitate the transport of lymph against hydrostatic pressure (76). Intraluminal valves like those in the great veins are present to prevent the backflow of lymph and divide the collecting lymphatic vessels into functional pumping units termed lymphangions $(77,78)$. By contracting, collecting lymphatic vessels force the lymph into the venous circulation at the sites of the lymphovenous valves.

FOXC2-calcineurin/NFATC1 signaling. Collecting lymphatics and their valves develop almost simultaneously (detected at approximately E14.5-E15.5 and E16.0, respectively) (79). As collecting lymphatic vessels mature, lymphatic capillary markers such as PROX1, VEGFR-3, and LYVE1 are downregulated in most LECs, ECM is deposited around the vessels, and SMCs begin to cover the vessels $(79,80)$. However, in lymphatic valve-forming cells, expression of PROX1 and FOXC2 remains elevated (79). Several 


\section{Table 4}

Loss-of-function phenotypes of genes associated with lymphatic vasculature development and disease

\begin{tabular}{|c|c|c|c|}
\hline Gene & Loss-of-function phenotype in animal models & $\begin{array}{l}\text { Related human } \\
\text { vascular disease }\end{array}$ & Reference(s) \\
\hline \multicolumn{4}{|c|}{ Defective blood and lymphatic vessel separation } \\
\hline Clec2 an & $\begin{array}{l}\text { Inactivation of this PDPN receptor results in defects similar to those reported for Pdpn } \\
\text { and SLP76; mutant embryos show blood-filled intestinal and mesenteric lymphatic vessels }\end{array}$ & & 68,69 \\
\hline Fiaf & Dilated and blood-filled lymphatic vessels in the intestine & & 74 \\
\hline Plcg2 & Blood-filled lymphatic vessels & & 117 \\
\hline Pdpn & Embryonic lethal at birth; lymphedema and dilated and blood-filled lymphatic vessels & & 56,61 \\
\hline Rac1 & $\begin{array}{c}\text { Conditional deletion results in embryonic lethality before } \\
\text { birth, edema, and blood-filled lymphatic vessels }\end{array}$ & & 73 \\
\hline SLP76 & Severe subcutaneous edema, peritoneal hemorrhage, and chylous ascites & & $59,60,68$ \\
\hline Syk & Severe subcutaneous edema and blood-filled lymphatic vessels. & & 59,69 \\
\hline \multicolumn{4}{|c|}{ Defective lymphatic vessel growth } \\
\hline Afadin & $\begin{array}{l}\text { Modulates RhoA; severe subcutaneous edema with severe disruption of } \\
\text { VE-cadherin-mediated cell-cell junctions in lymphatic vessels of the skin }\end{array}$ & & 27 \\
\hline Ang2 & $\begin{array}{l}\text { Subcutaneous edema, chylous ascites, lymphatic vessel } \\
\text { hypoplasia, and mispatterned lymphatic vessels in the mesentery }\end{array}$ & & 118 \\
\hline Aspp1 & $\begin{array}{l}\text { Null embryos exhibit subcutaneous edema, defective lymphatic } \\
\text { drainage, and mispatterned collecting lymphatic vessels }\end{array}$ & & 113 \\
\hline Cxadr & $\begin{array}{l}\text { Conditional deletion at E12.5 results in subcutaneous edema, hemorrhage, and } \\
\text { embryonic death with dilated subcutaneous lymphatic vessels that appear structurally } \\
\text { abnormal, exhibiting gaps and holes in LEC cell-cell junctions; blood-filled lymphatics } \\
\text { show defects in the separation of the blood and lymphatic vasculatures }\end{array}$ & & 28 \\
\hline Cx26 & $\begin{array}{l}\text { Conditional deletion in the ectoderm results in embryonic death before birth, } \\
\text { severe subcutaneous edema, and reduced dermal lymphatic capillary network }\end{array}$ & & 90 \\
\hline Integrin- $\beta 1$ & $\begin{array}{c}\text { Edema and hemorrhages; embryonic lethality; reduced LEC } \\
\text { numbers and LEC proliferation; smaller lymph sacs; complete lack of } \\
\text { dermal and mesenteric lymphatic vasculature at E15.5 }\end{array}$ & & 99 \\
\hline Nrp2 & Absence or severe reduction of small lymphatic vessels and capillaries & & 41 \\
\hline Ptpn14 & Lymphedema; lymphatic hyperplasia; interacts with VEGFR-3 & $\begin{array}{l}\text { Lymphedema-choanal } \\
\text { atresia syndrome }\end{array}$ & 119 \\
\hline Rasa1 & Hyperplasia, dilation, and leakage of lymphatic vessels and chylothorax & $\begin{array}{l}\text { Capillary and } \\
\text { arteriovenous malformation }\end{array}$ & 120 \\
\hline$T b x 1$ & $\begin{array}{l}\text { Regulates VEGFR3; conditional deletion in ECs results in embryonic } \\
\text { edema and postnatal lethality between } 2 \text { and } 4 \text { days after birth; } \\
\text { mice exhibit chylous ascites and lack of mesenteric lymphatic vessels }\end{array}$ & DiGeorge syndrome & 114,115 \\
\hline TGFBRI or TGFBRII & $\begin{array}{l}\text { Severe edema; blood-filled lymphatic vessels; reduced } \\
\text { lymphatic branching; aberrant lymphatic vessel network }\end{array}$ & & 110 \\
\hline Vezf1 & $\begin{array}{c}\text { Lymphatic hypervascularization, edema, and hemorrhaging } \\
\text { the jugular region of heterozygous embryos }\end{array}$ & & 121 \\
\hline
\end{tabular}

crucial signaling pathways are associated with the formation of collecting lymphatic vessels (Tables $2-4$ ). The best-characterized pathway is FOXC2/calcineurin/NFATC1 signaling, which is indispensable for both the maturation of collecting lymphatics and the formation and maintenance of lymphatic valves. The transcription factor Foxc2 was previously shown to be necessary for correct lymphatic patterning and mural cell recruitment during the maturation of collecting lymphatics (81). More recently, in Foxc2-knockout mice the primitive lymphatic plexus was shown to maintain high expression of capillary markers (e.g., PROX1, VEGFR-3, and LYVE1) without differentiating into functional collecting lymphatics and valves, as indicated by the backflow of lymph $(81,82)$. Intriguingly, point mutations in human FOXC2 are associated with lymphedema-distichiasis syndrome $(83,84)$, in which lymphatic and venous valves are defective (85). Genomewide ChIP-on-ChIP analysis showed that FOXC2 cooperates with the cardiac valve development transcription factor calcineurin/
NFATC1, which is present in LECs during the maturation of collecting lymphatic vessels $(82,86-88)$. Moreover, inducible deletion of the calcineurin regulatory subunit $\mathrm{Cnb1}$ at any mouse embryonic stage leads to defects in the formation of the lymphatic valve territory and the lymphatic valves themselves (79). Several studies have shown that gap junction proteins of the connexin family (CX26, CX37, and CX43) are important for lymphangiogenesis during development (refs. 89, 90, and Tables 2-4). For example, Cx37 knockout mice lack lymphatic valve-forming cells and have no lymphatic valves $(79,89)$, and mutations in CX47 are associated with primary lymphedema in humans $(91,92)$. Strikingly, in vitro flow analyses revealed that calcineurin/NFATC1 activation is markedly reduced when $C \times 37$ is depleted, and that PROX1, FOXC2, and oscillatory shear stress regulate the expression of Cx37 (79). Taken together, these results indicate that this pathway is crucial for the maturation of collecting lymphatic vessels and the formation and maintenance of lymphatic valves. 


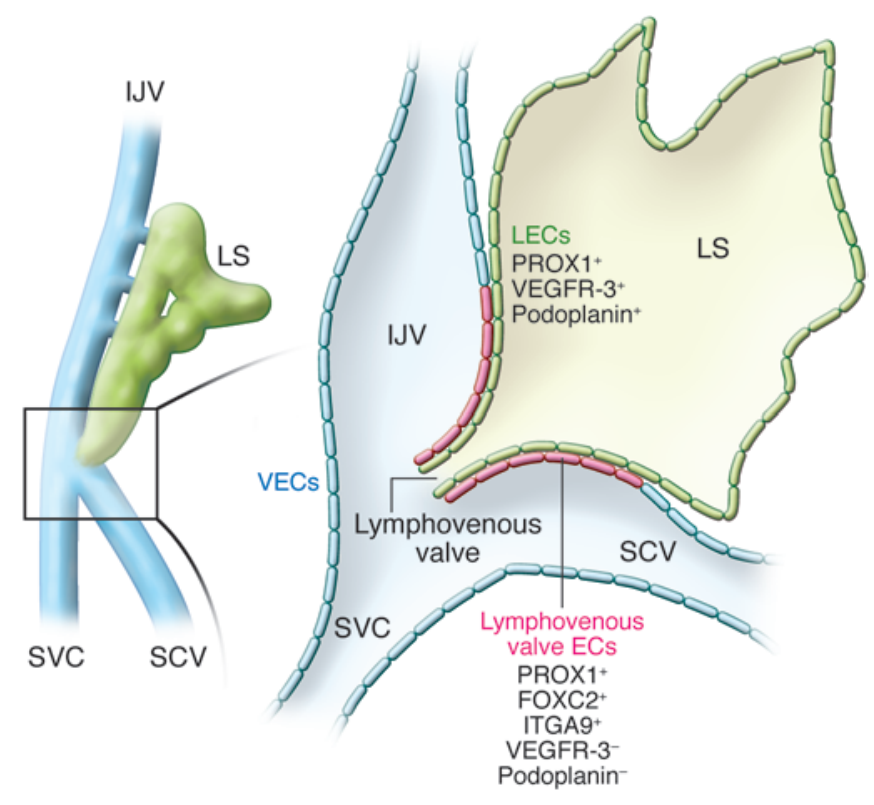

Figure 2

Diagrammatic representation of the lymphovenous valves. Although most PROX1-expressing LEC progenitors bud off from the veins, a small subpopulation remains and forms the lymphovenous valves at the junction of the jugular and subclavian veins (SCV). This unique population of ECs is negative for PDPN and VEGFR-3. Each of the valve's two leaflets has two layers of PROX1+ECs: an inner PROX1+/ PDPN ${ }^{+}$layer continuous with the lymph sac and an outer PROX $1^{+}$ PDPN- layer continuous with the veins. Left: The region of an E13.5 embryo in which the jugular and subclavian veins join to form the lymphovenous valves. Right: A frontal view of the boxed region shown at left. EJV, external jugular vein; IJV, internal jugular vein; LS, lymph sac; LV, lymphovenous valve; SVC, superior vena cava.

Reelin signaling. The reelin pathway is also important for the formation of collecting lymphatic vessels. Immunofluorescence studies detected reelin expression in LECs of collecting lymphatics and lymphatic capillaries. Interestingly, the reelin signal was intracellular in lymphatic capillaries and extracellular in collecting lymphatic vessels, especially near the valve regions, indicating that LECs of the collecting lymphatics secrete reelin into the ECM near the valves (80). Moreover, this LEC secretion of reelin protein is strongly associated with the migration and adherence of SMCs to collecting lymphatics. In reelin-mutant mice, the dermal collecting lymphatics are dilated and retain abnormally high expression of LYVE1, and SMC recruitment to collecting lymphatic vessels is reduced (80). The function of these vessels is also impaired in reelin knockout mice, whose collecting lymphatics are leaky and show a reduced rate of lymphatic flow in real-time imaging of indocyanine green dye uptake (Tables 2-4). Therefore, reelin serves an important and unique function in the formation of collecting lymphatics as an LEC-specific matrix molecule.

ECM components in lymphatic valve formation. As ECM deposition is a characteristic feature of collecting lymphatic vessels maturation, it is not surprising that ECM protein receptors are involved in development of the lymphatic vasculature. For example, many reports have described the function of integrins during lymphangiogenesis. Integrin signaling can promote the migration, proliferation, and survival of LECs (93-99). The integrin family contains eight $\alpha$ and $18 \beta$ subunits, which can form 24 integrin transmembrane heterodimers to mediate cell-cell and cell-ECM interactions (100). Specifically, integrin- $\alpha 9$ has fibronectin as its ligand, and its interaction with the ECM protein EMILIN1 plays a crucial role during lymphatic valve morphogenesis $(101,102)$. Integrin- $\alpha 9$ is highly expressed in mature and developing lymphatic valves, which are reported to be reduced in number and be morphologically abnormal in integrin- $\alpha 9$-deficient mice. The matrix core of the valves fails to form in these mice, leading to leakage and backflow of lymph (101). The finding of congenital chylothorax in human fetuses with mutations in the ITGA9 gene (103) is consistent with these reports.

Axonal guidance genes. It is well established that during blood vessel growth the endothelial tip cells and the axon growth cones are guided by common signaling cues. Many of these proteins also play significant roles in lymphangiogenesis (104). The axonal guidance genes semaphorin $3 \mathrm{~A}$ (Sema3a) and its receptors Nrp1 and plexin A1 (Plxna1) are expressed in collecting lymphatic vessels and lymphatic valves $(105,106)$. Sema3A is required for lymphatic valve formation. The valve area is significantly smaller in Sema3a $a^{-/-}$and Plxna1 $1^{-/-}$mice (106). Inhibition of SEMA3A/ NRP1 binding leads to irregularly shaped collecting lymphatic vessels, abnormally small lymphatic valves, and defects in postnatal lymph flow $(105,106)$. In addition to these morphological and functional problems, ectopic SMCs cover collecting lymphatic vessels and integrin- $\alpha 9$, and the integrin- $\alpha 9$ ligand FN1 is aberrantly expressed on lymphatic valve leaflets in the absence of the SEMA3A signal (105). These valvular defects result in impaired lymphatic flow (105). In summary, both integrin- $\alpha 9$ and SEMA3A-NRP1 signaling are required in later stages of valve development but do not affect early valve specification. Another axon guidance molecule, ephrin B2, a member of the Eph receptor tyrosine kinase family, has also been identified as an essential regulator of lymphatic development (107). That study demonstrated that the C-terminal PDZ interaction site of ephrin B2 is required to mediate the function of ephrin B2 in the formation of collecting lymphatic vessels and lymphatic valves, and hyperplastic collecting lymphatics and absence of valves were observed in ephrin B2 PDZ domain knockout mice (107). Similar to the Foxc2 and reelin deficiency, loss of ephrin B2 PDZ domain affected the specification of collecting lymphatic identity, as shown by an abnormally high level of expression of LYVE1 in the hyperplastic lymphatics (107).

Other important factors in lymphatic vessel and valve formation. BMP9, a ligand of the TGF- $\beta$ family type 1 receptor ALK1, was recently identified as a participant in the maturation of lymphatic capillaries, collecting lymphatic vessels, and lymphatic valves (ref. 108 and Tables 2-4). Bmp9-knockout mice exhibit abnormal patterning of both lymphatic capillaries and collecting lymphatic vessels, and the enlarged collecting lymphatic vessels retain LYVE1 expression, indicating that their maturation is affected. In primary cultured human LECs, BMP9 regulates LYVE1 expression through its receptor ALK1 (108). Moreover, pups lacking Bmp9 have a significantly reduced total number of valves, and therefore decreased lymph flow. Mechanistically, Bmp9 induces expression of FOXC2, CX37, ephrin B2, and NRP1 in an ALK1-dependent manner (108), consistent with the previous finding that ALK1 signaling regulates postnatal lymphatic vasculature patterning (109). Therefore, BMP9 is essential for development of the lymphatic capillaries and collecting vessels as well as for the 
formation of lymphatic valves. In addition to ALK1, conditional deletion of the TGF- $\beta$ receptors Tgfbr 1 and Tgfbr 2 in LECs leads to a severe reduction in lymphangiogenic sprouting (110).

AKT-mediated signaling plays an important role in development of the lymphatic vasculature (111). Akt1 $1^{-/-}$mice have smaller lymphatic capillaries, and their small collecting lymphatic vessels lack valves; however, valves are present in the large collecting lymphatic vessels. The collecting lymphatic vessels in $A k t 1^{-/-}$mice are enlarged in diameter and show abnormal SMC coverage and defective lymph flow (111).

It has recently been revealed that the planar cell polarity proteins CELSR1 and VANGL2 also participate in lymphatic valve formation (112). During valve leaflet morphogenesis, LECs undergo elongation, reorientation, and collective migration into the vessel lumen. Celsr1- or Vangl2-null mice lack valves because LECs fail to rearrange and adopt perpendicular orientation at valve initiation sites (112).

Finally, apoptosis-stimulating protein of p53 (ASPP1) knockout mice show subcutaneous edema and mispatterned lymphatic collecting vessels (113). Functional analysis has revealed impaired lymphatic drainage in Aspp $1^{-/-}$embryos. Although it has been reported that ASPP1 enhances apoptotic activity of p53, the function of ASPP1 in lymphatic vasculature development is independent of p53 (113).

\section{Conclusions}

Although our knowledge of developmental lymphangiogenesis has drastically improved, many interesting questions remain to be analyzed in the years to come. For example, what determines the number of LEC progenitors that will bud from the veins? What determines the location of the forming lymph sacs? Many key regulators of the maturation of collecting lymphatics and the formation of lymphatic valves have been identified; however, additional factors and signaling pathways are likely to participate in this process. In particular, it will be interesting to identify those factors essential for the formation of more specialized lymphatics, such those of mesenteric lymphatics or other specific organs. For example, Tbx1-deficient mice have hyperplastic lymphatic vessels in the heart, diaphragm, and skin, and they lack the entire gastrointestinal lymphatic vasculature (114). Interestingly, Tbx 1 is associated with DiGeorge syndrome; however, lymphatic defects so far have been reported just once in patients with this syndrome (115). It is likely that a better understanding of the morphogenetic process leading to the formation of the mature lymphatic network will facilitate the identification of additional functional roles of the lymphatic vasculature that, when defective, could lead to different pathological conditions. Also, it will be interesting to better understand the functional importance of EC fate plasticity does this reprogramming potential occur only under certain circumstances, such that blood and LECs can switch fates?

\section{Acknowledgments}

The authors want to thank Jennifer James, who created all the figures included in this Review. This work was supported by NIH grant R01-HL073402 and by the Leducq Foundation and the American Lebanese Syrian Associated Charities.

Address correspondence to: Guillermo Oliver, Department of Genetics, St. Jude Children's Research Hospital, 262 Danny Thomas Place, Memphis, Tennessee 38105, USA. Phone: 901.595.2697; Fax: 901.595.6035; E-mail: guillermo.oliver@stjude.org.

Ying Yang's present address is: University of Missouri, Bond Life Sciences Center, Columbia, Missouri, USA.
1. Sabin F. On the origin of the lymphatics system from the veins and the development of the lymph hearts and the thoracic duct in the pig. Am J Anat. 1902;1(3):367-389.

2. van der Putte SC. The early development of the lymphatic system in mouse embryos. Acta Morphol Neerl Scand. 1975;13(4):245-286.

3. Srinivasan RS, et al. Lineage tracing demonstrates the venous origin of the mammalian lymphatic vasculature. Genes Dev. 2007;21(19):2422-2432.

4. Yaniv K, Isogai S, Castranova D, Dye L, Hitomi J, Weinstein BM. Live imaging of lymphatic development in the zebrafish. Nat Med. 2006;12(6):711-716.

5. Yang Y, et al. Lymphatic endothelial progenitors bud from the cardinal vein and intersomitic vessels in mammalian embryos. Blood. 2012; 120(11):2340-2348.

6. Hagerling R, et al. A novel multistep mechanism for initial lymphangiogenesis in mouse embryos based on ultramicroscopy. EMBO J. 2013;32(5):629-644.

7. Wigle JT, Oliver G. Prox1 function is required for the development of the murine lymphatic system. Cell. 1999;98(6):769-778.

8. Wigle JT, et al. An essential role for Prox1 in the induction of the lymphatic endothelial cell phenotype. EMBO J. 2002;21(7):1505-1513.

9. Oliver G, Srinivasan RS. Endothelial cell plasticity: how to become and remain a lymphatic endothelial cell. Development. 2010;137(3):363-372.

10. Francois M, et al. Sox18 induces development of the lymphatic vasculature in mice. Nature. 2008; 456(7222):643-647.

11. Srinivasan RS, et al. The nuclear hormone receptor Coup-TFII is required for the initiation and early maintenance of Prox 1 expression in lymphatic endothelial cells. Genes Dev. 2010;24(7):696-707.

12. Lin FJ, Chen X, Qin J, Hong YK, Tsai MJ, Tsai SY.
Direct transcriptional regulation of neuropilin-2 by COUP-TFII modulates multiple steps in murine lymphatic vessel development. J Clin Invest. 2010; 120(5):1694-1707.

13. Davis RB, Curtis CD, Griffin CT. BRG1 promotes COUP-TFII expression and venous specification during embryonic vascular development. Development. 2013;140(6):1272-1281.

14. Zheng W, et al. Notch restricts lymphatic vessel sprouting induced by vascular endothelial growth factor. Blood. 2011;118(4):1154-1162.

15. Kang J, et al. An exquisite cross-control mechanism among endothelial cell fate regulators directs the plasticity and heterogeneity of lymphatic endothelial cells. Blood. 2010;116(1):140-150.

16. Murtomaki A, et al. Notch1 functions as a negative regulator of lymphatic endothelial cell differentiation in the venous endothelium. Development. 2013; 140(11):2365-2376.

17. Irrthum A, et al. Mutations in the transcription factor gene SOX18 underlie recessive and dominant forms of hypotrichosis-lymphedema-telangiectasia. Am J Hum Genet. 2003;72(6):1470-1478.

18. Deng Y, Atri D, Eichmann A, Simons M. Endothelial ERK signaling controls lymphatic fate specification. J Clin Invest. 2013;123(3):1202-1215.

19. Aoki Y, Niihori T, Narumi Y, Kure S, Matsubara Y. The RAS/MAPK syndromes: novel roles of the RAS pathway in human genetic disorders. Hum Mutat. 2008;29(8):992-1006.

20. Tidyman WE, Rauen KA. The RASopathies: developmental syndromes of Ras/MAPK pathway dysregulation. Curr Opin Genet Dev. 2009;19(3):230-236.

21 . Hong YK, et al. Prox 1 is a master control gene in the program specifying lymphatic endothelial cell fate. Dev Dyn. 2002;225(3):351-357.

22. Kim H, Nguyen VP, Petrova TV, Cruz M, Alitalo K,
Dumont DJ. Embryonic vascular endothelial cells are malleable to reprogramming via Prox 1 to a lymphatic gene signature. BMC Dev Biol. 2010;10:72.

23. Johnson NC, et al. Lymphatic endothelial cell identity is reversible and its maintenance requires Prox1 activity. Genes Dev. 2008;22(23):3282-3291.

24. Harvey NL, et al. Lymphatic vascular defects promoted by Prox 1 haploinsufficiency cause adult-onset obesity. Nat Genet. 2005;37(10):1072-1081.

25. Srinivasan RS, Oliver G. Prox 1 dosage controls the number of lymphatic endothelial cell progenitors and the formation of the lymphovenous valves. Genes Dev. 2011;25(20):2187-2197.

26. Yao LC, Baluk P, Srinivasan RS, Oliver G, McDonald DM. Plasticity of button-like junctions in the endothelium of airway lymphatics in development and inflammation. Am J Pathol. 2012;180(6):2561-2575.

27. Majima T, et al. An adaptor molecule afadin regulates lymphangiogenesis by modulating RhoA activity in the developing mouse embryo. PLoS One. 2013;8(6):e68134.

28. Mirza M, et al. Essential role of the coxsackieand adenovirus receptor (CAR) in development of the lymphatic system in mice. PLoS One. 2012; $7(5): \mathrm{e} 37523$.

29. Francois $M$, et al. Segmental territories along the cardinal veins generate lymph sacs via a ballooning mechanism during embryonic lymphangiogenesis in mice. Dev Biol. 2011;364(2):89-98.

30. Kuchler AM, Gjini E, Peterson-Maduro J, Cancilla B, Wolburg H, Schulte-Merker S. Development of the zebrafish lymphatic system requires VEGFC signaling. Curr Biol. 2006;16(12):1244-1248.

31. Karkkainen MJ, et al. Vascular endothelial growth factor $\mathrm{C}$ is required for sprouting of the first lymphatic vessels from embryonic veins. Nat Immunol. 2004;5(1):74-80. 
32. Jeltsch M, et al. Hyperplasia of lymphatic vessels in VEGF-C transgenic mice. Science. 1997; 276(5317):1423-1425.

33. Kukk E, et al. VEGF-C receptor binding and pattern of expression with VEGFR-3 suggests a role in lymphatic vascular development. Development. 1996; 122(12):3829-3837.

34. Zhang L, et al. VEGFR-3 ligand-binding and kinase activity are required for lymphangiogenesis but not for angiogenesis. Cell Res. 2010;20(12):1319-1331.

35. Irrthum A, Karkkainen MJ, Devriendt K, Alitalo $\mathrm{K}$, Vikkula M. Congenital hereditary lymphedema caused by a mutation that inactivates VEGFR3 tyrosine kinase. Am JHum Genet. 2000;67(2):295-301.

36. Karkkainen MJ, et al. Missense mutations interfere with VEGFR-3 signalling in primary lymphoedema. Nat Genet. 2000;25(2):153-159.

37. Karkkainen MJ, et al. A model for gene therapy of human hereditary lymphedema. Proc Natl Acad Sci US A. 2001;98(22):12677-12682.

38. Gordon K, et al. FLT4/VEGFR3 and Milroy disease: novel mutations, a review of published variants and database update. Hum Mutat. 2013;34(1):23-31.

39. Karpanen T, et al. Functional interaction of VEGF-C and VEGF-D with neuropilin receptors. FASEB J. 2006;20(9):1462-1472.

40. Xu Y, et al. Neuropilin-2 mediates VEGF-C-induced lymphatic sprouting together with VEGFR3. J Cell Biol. 2010;188(1):115-130.

41. Yuan L, et al. Abnormal lymphatic vessel development in neuropilin 2 mutant mice. Development. 2002;129(20):4797-4806

42. Hogan BM, et al. Ccbe 1 is required for embryonic lymphangiogenesis and venous sprouting. Nat Genet. 2009;41(4):396-398.

43. Facucho-Oliveira J, Bento M, Belo JA. Ccbe1 expression marks the cardiac and lymphatic progenitor lineages during early stages of mouse development. Int J Dev Biol. 2011;55(10-12):1007-1014.

44. Bos FL, et al. CCBE1 is essential for mammalian lymphatic vascular development and enhances the lymphangiogenic effect of vascular endothelial growth factor-C in vivo. Circ Res. 2011;109(5):486-491.

45. Alders $\mathrm{M}$, et al. Mutations in CCBE1 cause generalized lymph vessel dysplasia in humans. Nat Genet. 2009;41(12):1272-1274.

46. Connell F, et al. Linkage and sequence analysis indicate that CCBE1 is mutated in recessively inherited generalised lymphatic dysplasia. Hum Genet. 2010;127(2):231-241.

47. Connell FC, et al. CCBE1 mutations can cause a mild, atypical form of generalized lymphatic dysplasia but are not a common cause of non-immune hydrops fetalis. Clin Genet. 2012;81(2):191-197.

48. Fritz-Six KL, Dunworth WP, Li M, Caron KM. Adrenomedullin signaling is necessary for murine lymphatic vascular development. J Clin Invest. 2008; 118(1):40-50.

49. Gordon EJ, Rao S, Pollard JW, Nutt SL, Lang RA, Harvey NL. Macrophages define dermal lymphatic vessel calibre during development by regulating lymphatic endothelial cell proliferation. Development. 2010;137(22):3899-3910.

50. D'Amico G, Korhonen EA, Waltari M, Saharinen P, Laakkonen P, Alitalo K. Loss of endothelial Tie1 receptor impairs lymphatic vessel development-brief report. Arterioscler Thromb Vasc Biol. 2010; 30(2):207-209.

51. Qu X, Tompkins K, Batts LE, Puri M, Baldwin S. Abnormal embryonic lymphatic vessel development in Tie1 hypomorphic mice. Development. 2010; 137(8):1285-1295

52. Kulkarni RM, Greenberg JM, Akeson AL. NFATc1 regulates lymphatic endothelial development. Mech Dev. 2009;126(5-6):350-365.

53. Lim KC, et al. Conditional Gata2 inactivation results in HSC loss and lymphatic mispatterning. J Clin Invest. 2012;122(10):3705-3717.
54. Ostergaard P, et al. Mutations in GATA2 cause primary lymphedema associated with a predisposition to acute myeloid leukemia (Emberger syndrome). Nat Genet. 2011;43(10):929-931.

55 . Kazenwadel J, et al. Loss-of-function germline GATA2 mutations in patients with MDS/AML or MonoMAC syndrome and primary lymphedema reveal a key role for GATA2 in the lymphatic vasculature. Blood. 2012;119(5):1283-1291.

56. Uhrin P, et al. Novel function for blood platelets and podoplanin in developmental separation of blood and lymphatic circulation. Blood. 2010; 115(19):3997-4005.

57. Oliver G. Lymphatic vasculature development. Nat Rev Immunol. 2004;4(1):35-45.

58. Tammela T, Alitalo K. Lymphangiogenesis: Molecular mechanisms and future promise. Cell. 2010 140(4):460-476.

59. Abtahian F, et al. Regulation of blood and lymphatic vascular separation by signaling proteins SLP-76 and Syk. Science. 2003;299(5604):247-251.

60. Sebzda E, et al. Syk and Slp-76 mutant mice reveal a cell-autonomous hematopoietic cell contribution to vascular development. Dev Cell. 2006;11(3):349-361.

61. Schacht V, et al. T1alpha/podoplanin deficiency disrupts normal lymphatic vasculature formation and causes lymphedema. EMBO J. 2003; 22(14):3546-3556.

62. Cheng AM, Rowley B, Pao W, Hayday A, Bolen JB, Pawson T. Syk tyrosine kinase required for mouse viability and B-cell development. Nature. 1995; 378(6554):303-306

63. Turner $M$, et al. Perinatal lethality and blocked B-cell development in mice lacking the tyrosine kinase Syk. Nature. 1995;378(6554):298-302.

64. Okuda T, van Deursen J, Hiebert SW, Grosveld G, Downing JR. AML1, the target of multiple chromosomal translocations in human leukemia, is essential for normal fetal liver hematopoiesis. Cell. 1996; 84(2):321-330

65. Clements JL, et al. Requirement for the leukocyte-specific adapter protein SLP-76 for normal T cell development. Science. 1998;281(5375):416-419.

66. Pivniouk V, Tsitsikov E, Swinton P, Rathbun G, Alt FW, Geha RS. Impaired viability and profound block in thymocyte development in mice lacking the adaptor protein SLP-76. Cell. 1998;94(2):229-238.

67. Carramolino L, Fuentes J, Garcia-Andres C, Azcoitia V, Riethmacher D, Torres M. Platelets play an essential role in separating the blood and lymphatic vasculatures during embryonic angiogenesis. Circ Res. 2010;106(7):1197-1201.

68. Bertozzi CC, et al. Platelets regulate lymphatic vascular development through CLEC-2-SLP-7 6 signaling. Blood. 2010;116(4):661-670.

69. Finney BA, et al. CLEC-2 and Syk in the megakaryocytic/platelet lineage are essential for development. Blood. 2012;119(7):1747-1756

70. Kato Y, et al. Molecular identification of Aggrus/ T1alpha as a platelet aggregation-inducing factor expressed in colorectal tumors. J Biol Chem. 2003; 278(51):51599-51605.

71. Kaneko M, Kato Y, Kunita A, Fujita N, Tsuruo T, Osawa M. Functional sialylated O-glycan to platelet aggregation on Aggrus (T1alpha/Podoplanin) molecules expressed in Chinese hamster ovary cells. J Biol Chem. 2004;279(37):38838-38843.

72. Osada M, et al. Platelet activation receptor CLEC2 regulates blood/lymphatic vessel separation by inhibiting proliferation, migration, and tube formation of lymphatic endothelial cells. J Biol Chem. 2012;287(26):22241-22252

73. D'Amico G, et al. Regulation of lymphatic-blood vessel separation by endothelial Rac1. Development. 2009;136(23):4043-4053.

74. Backhed F, Crawford PA, O'Donnell D, Gordon JI. Postnatal lymphatic partitioning from the blood vasculature in the small intestine requires fasting-induced adipose factor. Proc Natl Acad Sci US A. 2007;104(2):606-611.

75. Jurisic G, Detmar M. Lymphatic endothelium in health and disease. Cell Tissue Res. 2009;335(1):97-108.

76. Scallan JP, Davis MJ. Genetic removal of basal nitric oxide enhances contractile activity in isolated murine collecting lymphatic vessels. J Physiol. 2013; 591(Pt 8):2139-2156.

77. Sacchi G, Weber E, Agliano M, Raffaelli N, Comparini $\mathrm{L}$. The structure of superficial lymphatics in the human thigh: precollectors. Anat Rec. 1997; 247(1):53-62.

78. Casley-Smith JR. The fine structure and functioning of tissue channels and lymphatics. Lymphology. 1980;13(4):177-183.

79. Sabine A, et al. Mechanotransduction, PROX1, and FOXC2 cooperate to control connexin 37 and calcineurin during lymphatic-valve formation. Dev Cell. 2012;22(2):430-445.

80. Lutter S, Xie S, Tatin F, Makinen T. Smooth muscle-endothelial cell communication activates Reelin signaling and regulates lymphatic vessel formation. J Cell Biol. 2012;197(6):837-849.

81. Petrova TV, et al. Defective valves and abnormal mural cell recruitment underlie lymphatic vascular failure in lymphedema distichiasis. Nat Med. 2004; 10(9):974-981.

82. Norrmen C, et al. FOXC2 controls formation and maturation of lymphatic collecting vessels through cooperation with NFATc1. J Cell Biol. 2009; 185(3):439-457.

83. Fang J, et al. Mutations in FOXC2 (MFH-1), a forkhead family transcription factor, are responsible for the hereditary lymphedema-distichiasis syndrome. Am J Hum Genet. 2000;67(6):1382-1388.

84. Brice G, et al. Analysis of the phenotypic abnormalities in lymphoedema-distichiasis syndrome in 74 patients with FOXC2 mutations or linkage to 16q24. J Med Genet. 2002;39(7):478-483.

85. Mellor RH, et al. Mutations in FOXC2 are strongly associated with primary valve failure in veins of the lower limb. Circulation. 2007;115(14):1912-1920.

86. Chang CP, et al. A field of myocardial-endocardial NFAT signaling underlies heart valve morphogenesis. Cell. 2004;118(5):649-663.

87. de la Pompa JL, et al. Role of the NF-ATc transcription factor in morphogenesis of cardiac valves and septum. Nature. 1998;392(6672):182-186.

88. Ranger AM, et al. The transcription factor NF-ATc is essential for cardiac valve formation. Nature. 1998; 392(6672):186-190

89. Kanady JD, Dellinger MT, Munger SJ, Witte MH, Simon AM. Connexin 37 and Connexin43 deficiencies in mice disrupt lymphatic valve development and result in lymphatic disorders including lymphedema and chylothorax. Dev Biol. 2011;354(2):253-266

90. Dicke N, et al. Peripheral lymphangiogenesis in mice depends on ectodermal connexin-26 (Gjb2). J Cell Sci. 2011;124(pt 16):2806-2815.

91. Ferrell RE, et al. GJC2 missense mutations cause human lymphedema. Am J Hum Genet. 2010; 86(6):943-948.

92. Ostergaard P, et al. Rapid identification of mutations in GJC2 in primary lymphoedema using whole exome sequencing combined with linkage analysis with delineation of the phenotype. J Med Genet. 2011;48(4):251-255.

93. Huang XZ, et al. Fatal bilateral chylothorax in mice lacking the integrin alpha9beta1. Mol Cell Biol. 2000; 20(14):5208-5215.

94. Mishima K, et al. Prox 1 induces lymphatic endothelial differentiation via integrin $\alpha 9$ and other signaling cascades. Mol Biol Cell. 2007; 18(4):1421-1429

95. Hong YK, et al. VEGF-A promotes tissue repair-associated lymphatic vessel formation via VEGFR-2 and the $\alpha 1 \beta 1$ and $\alpha 2 \beta 1$ integrins. FASEB J. 2004; 18(10):1111-1113. 
96. Zhang X, Groopman JE, Wang JF. Extracellular matrix regulates endothelial functions through interaction of VEGFR-3 and integrin $\alpha 5 \beta 1$. J Cell Physiol. 2005;202(1):205-214.

97. Dietrich $\mathrm{T}$, et al. Inhibition of inflammatory lymphangiogenesis by integrin $\alpha 5$ blockade. $A m J$ Pathol. 2007;171(1):361-372.

98. Okazaki T, et al. $\alpha 5 \beta 1$ Integrin blockade inhibits lymphangiogenesis in airway inflammation. $A m J$ Pathol. 2009;174(6):2378-2387.

99. Planas-Paz L, Strilic B, Goedecke A, Breier G, Fassler R, Lammert E. Mechanoinduction of lymph vessel expansion. EMBO J. 2012;31(4):788-804.

100.Hynes RO. Integrins: bidirectional, allosteric signaling machines. Cell. 2002;110(6):673-687.

101.Bazigou $\mathrm{E}$, et al. Integrin- $\alpha 9$ is required for fibronectin matrix assembly during lymphatic valve morphogenesis. Dev Cell. 2009;17(2):175-186.

102. Danussi C, et al. The EMILIN1/ $\alpha 9 \beta 1$ integrin interaction is crucial in lymphatic valve formation and maintenance. Mol Cell Biol. 2013;33(22):4381-4394.

103. Ma GC, et al. A recurrent ITGA9 missense mutation in human fetuses with severe chylothorax: possible correlation with poor response to fetal therapy. Prenat Diagn. 2008;28(11):1057-1063.

104.Larrivee B, Freitas C, Suchting S, Brunet I, Eichmann A. Guidance of vascular development: lessons from the nervous system. Circ Res. 2009; 104(4):428-441.
105.Jurisic G, et al. An unexpected role of semaphorin3a-neuropilin-1 signaling in lymphatic vessel maturation and valve formation. Circ Res. 2012; 111(4):426-436.

106. Bouvree K, et al. Semaphorin3A, Neuropilin-1, and PlexinA1 are required for lymphatic valve formation. Circ Res. 2012;111(4):437-445.

107. Makinen T, et al. PDZ interaction site in ephrinB2 is required for the remodeling of lymphatic vasculature. Genes Dev. 2005;19(3):397-410.

108. Levet S, et al. Bone morphogenetic protein 9 (BMP9) controls lymphatic vessel maturation and valve formation. Blood. 2013;122(4):598-607.

109. Niessen K, Zhang G, Ridgway JB, Chen H, Yan M. ALK1 signaling regulates early postnatal lymphatic vessel development. Blood. 2010;115(8):1654-1661.

110.James JM, Nalbandian A, Mukouyama YS. TGFbeta signaling is required for sprouting lymphangiogenesis during lymphatic network development in the skin. Development. 2013;140(18):3903-3914.

111. Zhou F, et al. Akt/Protein kinase B is required for lymphatic network formation, remodeling, and valve development. Am J Pathol. 2010;177(4):2124-2133.

112. Tatin F, et al. Planar cell polarity protein celsr 1 regulates endothelial adherens junctions and directed cell rearrangements during valve morphogenesis. Dev Cell. 2013;26(1):31-44.

113. Hirashima M, Sano K, Morisada T, Murakami K, Rossant J, Suda T. Lymphatic vessel assembly is impaired in Aspp1-deficient mouse embryos. Dev Biol. 2008;316(1):149-159.

114. Chen $\mathrm{L}$, et al. Tbx1 regulates Vegfr 3 and is required for lymphatic vessel development. J Cell Biol. 2010; 189(3):417-424.

115.Yagi $\mathrm{H}$, et al. Role of TBX1 in human del22q11.2 syndrome. Lancet. 2003;362(9393):1366-1373.

116. Joukov V, et al. A novel vascular endothelial growth factor, VEGF-C, is a ligand for the Flt4 (VEGFR-3) and KDR (VEGFR-2) receptor tyrosine kinases. EMBO J. 1996;15(7):1751.

117. Ichise $\mathrm{H}$, Ichise $\mathrm{T}$, Ohtani $\mathrm{O}$, Yoshida $\mathrm{N}$. Phospholipase Cgamma2 is necessary for separation of blood and lymphatic vasculature in mice. Development. 2009;136(2):191-195.

118. Gale NW, et al. Angiopoietin-2 is required for postnatal angiogenesis and lymphatic patterning, and only the latter role is rescued by Angiopoietin-1. Dev Cell. 2002;3(3):411-423.

119.Au AC, et al. Protein tyrosine phosphatase PTPN14 is a regulator of lymphatic function and choanal development in humans. Am J Hum Genet. 2010; 87(3):436-444.

120. Lapinski PE, et al. RASA1 maintains the lymphatic vasculature in a quiescent functional state in mice. J Clin Invest. 2012;122(2):733-747.

121. Kuhnert F, et al. Dosage-dependent requirement for mouse Vezf 1 in vascular system development. Dev Biol. 2005;283(1):140-156. 